\title{
A Conceptualist View in the Metaphysics of Species
}

\author{
Ciro De Florio and and Aldo Frigerio
}

The species concept is one of the central concepts in biological science. Although modern systematics speculates about the existence of a complex hierarchy of nested taxa, biological species are considered particularly important for the active role they play in evolution. However, neither theoretical biologists nor philosophers of biology have come to an agreement about what a species is. In this chapter, we address two questions pertaining to biological species: (1) are they individuals or universals? and (2) are they bona fide or fiat entities? In section The Species-asIndividuals View, we illustrate the reasons that have led many scholars to support the view that species are individuals. In the next two sections, we show that the relational concepts of species - on which the species-as-individuals view is based provide neither necessary nor sufficient conditions for species membership. This seriously undermines the species-as-individuals view. In the section A Conceptualist Model for the Metaphysics of Species, we advance the proposal that species are fiat concepts (and thus, universal entities partially dependent on the human mind) carved in a multi-dimensional space representing the properties that the biological organisms possess. The final section concludes.

\section{The species-as-individuals view}

In the Aristotelian tradition, species are regarded as kinds, that is, as universal entities, predicated of individual substances that characterize their essence (for instance, the essence of being a dog). An individual substance (consider our cat Kurt as an example) instantiates a natural kind. The existence of the natural kind 'cat' depends on the existence of particular cats; however, the fact that Kurt 
lacks gills, for example, depends on his essence - his being an instance of the kind 'cat'. In this view, the offspring of a couple of organisms belonging to a species can diverge from their ancestors only by contingent characters. Essential and defining characters must remain the same through generations. Therefore, a species is defined as the set of traits that are regarded as sufficient and necessary for being a member of that species.

Darwin's theory of evolution has provided a framework in which the Aristotelian concept of biological species seems odd for many reasons. In Darwin's evolutionary view, in principle, every character of an organism can disappear in its offspring. Moreover, the distinction between defining and contingent characters disappears; every character can change, and none is necessarily constant through generations. When a character changes, it is difficult to decide whether it was a defining character of the species (so that the species evolved into another one) or whether it was contingent (so that the species remained the same). Every character is not stable in principle; therefore, the distinction between defining and contingent characters is not easy to trace. In the evolutionary framework, it is difficult to define a species as a set of necessary and sufficient traits because every trait can vary in an almost limitless way, and none seems to be essential.

Therefore, some alternative definitions of biological species have been formulated, and these are not grounded in the characters possessed by singular organisms but rather in the relations among members of the same species. In particular, two kinds of relations have received attention: interbreeding relations and genealogical relations among organisms. Interbreeding relations are at the basis of the biological concept of species. ${ }^{1}$ According to this concept, a species is a group of individuals that can breed together and are reproductively isolated from other organisms. ${ }^{2}$ The gene flow among members of the same species and the lack of gene flow with members of other species contribute to preserving the genetic pool of the species as relatively constant by recombining the genes of deviant individuals with those of conspecific individuals and protecting the genetic pool from the introgression of genes belonging to individuals of other species. In this way, the most favourable combinations of genes are preserved, and the production of too many disharmonious, incompatible gene combinations is prevented.

Another concept based not on the intrinsic properties of the members but on their relationships is the phylogenic concept. The basic idea is that an individual is a member of the species $S$ only if it descends from another individual of $S$. However, we cannot speak of a unique phylogenetic concept of species but rather a family of concepts related to each other but having remarkable differences. In particular, a phylogenetic concept is incomplete unless it is not specified 
when a speciation episode occurs, that is, when an individual descending from a member of $S$ is no longer a member of $S$. If this is not specified, the definition has the consequence that every descendant of an individual belonging to $S$ is a member of $S$, and thus, that speciation is impossible. In this framework, the arising of a new species can be indicated either by the reproductive isolation of a community of individuals from the other descending members ${ }^{3}$ or by the appearance of a new particular trait that is not present in the ancestors of an individual. ${ }^{4}$

These relational concepts of species favoured a new ontological view of species that are no longer regarded as abstract kinds instantiated by singular organisms but as complex individuals composed of singular organisms. As Ghiselin (1974) argues, species are similar to firms. To decide whether a person is employed at a certain firm, we do not have to observe his or her features but rather the relations between him or her and the other employees of that firm. Consequently, a firm is not a species instantiated by its employees - it is a complex individual whose employees are parts linked by some relation. Indeed, the unity of the firm is due to the relations among employees. The same can be said about species. In order to evaluate whether an individual belongs to a biological species, its features are merely an indication. What is decisive is the relations between the individual and the other members of the species. It is, therefore, natural to consider a species as a complex individual whose cohesion is determined by the relations among its members. Besides firms, biological species can be compared to other complex individuals whose unity is due to the relations among their members, such as musical bands, associations or families. ${ }^{5}$

To conceive of species as individuals has further advantages. According to the theory of evolution, biological species have a temporal beginning and a temporal end, evolve and change. Abstract entities (such as universals) are usually conceived as timeless and, thus, as entities that have no beginning and no end and cannot undergo changes and transformations. Many scholars have concluded that biological species cannot be kinds and that every attempt to deny this was anti-evolutionist and fixist because it would have implied the negation of the thesis that species evolve. A natural conclusion is that species are individuals because, unlike universals, individuals can have a temporal beginning, can have a temporal end and can change. Peter Simons sums up the question as follows:

Modern evolutionary biology is based on populations of organisms. In this respect it is bottom-up or nominalistic, since populations are particular, not universal. It constitutes a definitive rejection of the platonistic conception 
of biological kinds as ideal archetypes initiated by Plato, retained in modified form by Aristotle, and perpetuated by scholasticism, Linnæus, Goethe, Cuvier, and Owen. The gradual acceptance of evolution following Lamarck and the subsequent explanation of its mechanism by Darwin and Wallace mean that biologists can no longer sustain a top-down or typological conception of what constitutes a species. As to what species are, even under a single conception, theorists differ. The common-sense idea is that they are certain pluralities or collections of organisms, delimited by their interrelations. (Simons 2013, p. 278)

It is interesting to note that even a friend of natural kinds, Jonathan Lowe, distinguishes the notion of biological species - which he believes to be a complex and collective individual - from that of natural kind, which is a metaphysical category having to do with the structure of reality:

But perhaps we need to make a distinction, which can best be brought out by analogy with a related case, that of biological species. These too are said to come into existence and undergo change - indeed, that they do so is crucial to the theory of evolution. How then can species names denote universals, which are abstract entities and so on the present proposal timeless? The solution is to distinguish between biological species, which are concrete particulars consisting at any time of the mereological sum of their currently existing members (individual tigers or individual oaks), and biological sorts or kinds, which are universals instantiated by the members of those species. (Lowe 1998, p. 75)

To summarize, the naive interpretation of the concept of biological species as natural kind has been substantially revised in light of the theory of evolution. However, we will see in the next two sections that the relational concepts of species fail in many respects, and this seriously undermines the species-asindividuals view.

\section{The biological concept provides neither necessary nor sufficient conditions}

In this and the next sections, we show that relational criteria provide neither necessary nor sufficient conditions for being a member of a species. Consequently, any biological taxonomy must, at least in part, turn to the similarity among organisms in their classification. Thus, one of the main arguments for regarding species as complex individuals fails. If relations are neither necessary nor sufficient to ascribe an individual to a species and we need to rely on similarity among organisms, the analogy between biological species and complex 
individuals such as firms or musical bands must be dismissed. Let us consider the biological concept first. We will show that it provides neither necessary nor sufficient conditions for being a member of a species.

\section{Not necessary}

Critics of the biological concept often highlight that it can be applied only to sexually reproducing organisms (Vrba 1985, Templeton 1989). This is a severe restriction, because most organisms on Earth are asexual but seem to display the same patterns of phenotypic cohesion within and discontinuity between species as do sexual species. Taxonomic practice has always subdivided the asexual world into easily defined biological taxa, as it has always done with the sexual world. Advocates of the biological concept can react in three ways to this restriction: they can claim that asexual organisms are members of no species (Mayr 1987); they can affirm that every asexual organism is the unique member of its own species since it is reproductively isolated from every other organism (Ghiselin 1974) or they can hold that a concept of species different from the biological concept should be applied to asexual organisms (Ereshefsky 2001). The first two solutions are hopeless in light of the fact that asexual organisms can be classified in species as easily as sexual organisms. Thus, the only way out seems to be maintaining that interbreeding relations are a necessary condition for being a member of a species for just a portion of the biological world: the sexual one.

However, this solution does not solve all the problems of the biological concept. Ehrlich and Raven (1969) argue that many conspecific sexual populations have little or no gene flow between them, particularly in plants, due to the physical distance between them. Moreover, 'selection can override the effects of gene flow' (p. 64) so that the cohesive force binding species together is for the most part 'similar selective regimes' (p. 63). The biological concept would overrate the importance of the interbreeding relations as a mechanism for preserving the unity of the species, which, on the contrary, would be largely caused by environmental factors. Ehrlich and Raven's thesis is questioned by Ridley (1993), who states that it is not clear if selection is more important than gene flow; the two are not necessarily mutually exclusive, and, depending on the situation, one might be more important than the other. He concludes that 'the evidence ... hardly warrants any firm conclusion' (p. 399) but that 'selection and genetic flow are probably not usually opposed forces in nature' (p. 397). Be that as it may, genetic flow is not a unique cohesive mechanism in sexual species, and perhaps it is not even the most important. 
There is another, more serious, problem with the biological concept - the members of a species $S$ that cannot mate with the other members of $S$ because of malformation or malfunctioning of the reproductive system, or sterility. If potential interbreeding were a necessary condition for being a member of a species, these organisms should be judged as non-members of the species $\mathrm{S}$. This is an unacceptable conclusion. Therefore, potential interbreeding among conspecific members is not a necessary condition for being a member of a species, even if the species is a sexual one. Could advocates of the biological concept appeal, as they often do, ${ }^{6}$ to particular isolating mechanisms to solve this problem? These mechanisms are of several kinds and are usually divided in two classes, 'namely mechanisms preventing the appearance of hybrid offspring (incompatibility of the parental forms), and mechanisms making the hybrids sterile and, consequently, incapable of propagating further (hybrid sterility)' (Dobzhansky 1935, p. 350). One could maintain that the members of S that are reproductively isolated from the other members of $S$ are isolated for mechanisms that are not typical of S. In other words, the isolated mechanisms that characterize $S$ are not those mechanisms that isolate the members of $S$ that cannot mate and reproduce with other members of $S$. However, since the malformed and sterile members of $S$ share the particular isolating mechanisms that reproductively isolate the species $\mathrm{S}$ from other species, they can be considered members of $\mathrm{S}$. Nevertheless, this answer is very risky for advocates of the biological concept. It seems to imply that being a member of $S$ means to possess a particular isolating mechanism (e.g. particular forms of genitalia that prevent mating with organisms of other species, a particular structure of chromosomes that prevent meiosis with chromosomes of different species). These isolating mechanisms are structural properties of organisms, not relational properties. Then, species membership would be determined by the possession of certain structural properties. The biological concept would not be a relational concept but would be grounded in the possession of some intrinsic properties of the members of a species. This would be against the intents of the advocates of the biological concept, who have always claimed that relationships - and not intrinsic properties - determine the membership to a species.

\section{Not sufficient}

Many species, especially among plants, ${ }^{7}$ are not reproductively isolated but can cross-breed with other species, giving rise to fertile and viable hybrids. An oftenmentioned case is that of oaks, a genus with more than 400 species that hybridize 
each other, creating new, fertile species. The new species can then hybridize with the parent species but remain distinct from them for long periods of time because of geographic barriers and a low rate of hybridization among the species (Burger 1975, Van Valen 1976). The frequency of the hybridization phenomena among different species shows that potential interbreeding is not a sufficient condition for being of the same species.

Another phenomenon that leads to the same conclusion is that interbreeding relationships are not transitive; population A can easily cross-breed with population $B$, and population $B$ can interbreed with population $C$, but this does not mean that A can cross-breed with C. A particular case of this phenomenon is that of the ring species. Ring species are continuously connected chains of races of a species that surround a geographic region than cannot be occupied because it is a mountain massif, ocean or polar region. Therefore, they show a ring-shaped distribution around the inhospitable region. At the endpoints of this geographic ring, the most distant populations encounter each other secondarily. Here, they are no longer genetically compatible and are unable to cross-breed. Although they still belong to the same species, they have become genetically different because their common ancestor dates back far into the past and the mutual exchange of alleles has become scarce or has ceased entirely. A good example of a ring species is the greenish warbler (Phylloscopus trochiloides), a passerine that spread from the south of the Tibetan Plateau to both the western and eastern sides of the plateau. Today, the Tibetan Plateau is surrounded by several races of greenish warblers that are all reproductively connected to each other. On the northern side of the Tibetan Plateau, however, the races from the west and the east encounter each other; here, they are mutually genetically incompatible. Between the two races in the contact zone, gene exchanges no longer occur (Irwin, Bensch and Price 2001). In many cases of ring species, the different races or sub-species end up being considered different species that can cross-breed with the species that are geographically close but not with those that are geographically distant. Again, potential cross-breeding does not imply being of the same species.

\section{The phylogenic concepts provide neither necessary nor sufficient conditions}

In this section, we will argue that phylogenic concepts provide neither necessary nor sufficient conditions for being a member of a species. 


\section{Not sufficient}

The proponents of phylogenetic concepts admit that these concepts do not provide sufficient conditions for species membership. The reason for this can be easily grasped - all living organisms likely descend from a unique organism or from a few organisms that lived some billions of years ago, but they obviously do not belong to the same species. Thus, in some cases, some organisms that descend from a member of the species $S$ are not members of $S$. This happens when a speciation event occurs. Therefore, a phylogenetic concept is incomplete unless it is not specified when a speciation episode occurs, that is, when an individual descending from a member of $S$ is no longer a member of $S$. If this is not specified, the definition has the consequence that every descendant of an individual belonging to $S$ is a member of $S$, and thus, that speciation is impossible. Phylogenetic lineage is not a sufficient criterion for species membership.

The issue is that the conditions that the advocates of the phylogenetic concepts add require that one either belongs to the same interbreeding community (which, as we have seen, is not a sufficient criterion) or possesses some phenotypic or genotypic characteristics. If this second route is followed, the criterion for species membership is not purely relational anymore; rather, it is based also on some intrinsic properties, that is, on the resemblance among members of the species.

\section{Not necessary}

The proponents of the phylogenetic concepts insist on the necessity of belonging to the same lineage for being a member of a species. Hence, Hull (1978) offered a thesis in which an extinct species cannot reappear again. When an individual has ceased to exist, it cannot begin to exist again: 'If a species evolved which was identical to a species of pterodactyl save origin, it would still be a new, distinct species' (p. 349). Since the two lineages have two different origins, they must be considered two different species. Modern taxonomic practices tend to follow the origin criterion for organizing the taxa to which biological organisms belong. It often happens that a certain taxon is moved from a superordinate taxon to another one in order to obtain only monophyletic taxa. ${ }^{8}$

However, the thesis that phylogenetic concepts provide necessary conditions can be put into question. Consider the following thought experiment. Suppose that on a distant planet, environmental conditions very similar to Earth's allow for the evolution of organisms that are genetically and phenotypically 
indistinguishable from terrestrial lions. Moreover, suppose that some of these animals are brought to Earth and begin to cross-breed with terrestrial lions. At a certain point, one cannot know whether an organism comes from the Twin Earth planet, from Earth or from a hybridization of the two races. ${ }^{9}$ According to the phylogenic concepts, terrestrial lions and twin lions are two different species, and the organisms descending from cross-breeds are hybrids of the two species. Moreover, the hybrids that cross-breed with the parent species give rise to new species. We would have several species without any possibility (genetic tests, morphological measurements, anatomical and physiological studies) to assign any individual to the species that are supposed to exist. This is an odd result.

Let us consider another thought experiment. Suppose that progresses in genetic engineering allow for the production of artificial life. Moreover, suppose that laboratory A produces a new life form starting from a certain genetic structure. Laboratory B buys the patent from laboratory A and produces organisms starting from the same genetic structure. Again, these two kinds of organisms are not distinguishable, both from genotypic and phenotypic points of view. Suppose that several laboratories produce this kind of organism and that they are scattered over environments. If sexual, they can freely interbreed. Again, according to the philological concepts, the organisms built in different laboratories are different species because they have different origins and belong to different lineages. When they cross-breed, they give rise to hybrids. But again, there is no possibility to distinguish the individuals when they are scattered in the environment. There is no test for establishing whether an organism originates from laboratory A rather than laboratory $\mathrm{B}$, whether it is a hybrid between the lineages of laboratory A and laboratory B, or whether it is a hybrid between an organism of this kind and an organism coming from laboratory A and so forth.

Thought experiments have never been very popular among philosophers of biology. Perhaps the reason for this is that biology is a science more empirical than physics, where thought experiments are very common. ${ }^{10}$

However, it is not possible to dismiss arguments of this kind simply on the ground that thought experiments are irrelevant in biology because there are actually very similar cases. Furthermore, these cases are very numerous. Many species hybridize and give rise to other species. There could be many hybridization events between two species $\mathrm{A}$ and $\mathrm{B}$ that are separate in time and space. They can give rise to several lineages of a species $\mathrm{C}$ that can also be separate in time and space. Some of these lineages can go extinct. Others can evolve in a parallel and independent way if they are subject to similar selective 
regimes. However, it is difficult to deny that these different lineages belong to the same species, although they have different origins. Furthermore, a hybridization episode between $\mathrm{A}$ and $\mathrm{B}$ can give rise to a lineage of the species $\mathrm{C}$, which can go extinct. Afterward, another hybridization episode can give rise to another lineage of $\mathrm{C}$. Thus, $\mathrm{C}$ is a species that went extinct and then began to exist again. Similar situations are probably very common among allopolyploid plants ${ }^{11}$ (i.e. plants in which homologous chromosomes are more than two and descend from different species), given the high number of allopolyploid species among angiosperms.

More rarely, two or more different and isolated populations of a species A, if subject to very similar selection regimes and to very similar environmental conditions, can separately evolve into a species B in parallel ways. In this case, different lineages of $\mathrm{B}$ have different origins. An actual example is the threespine stickleback. These fish live in different lakes in British Columbia and have followed very similar but independent evolutionary paths, which have led to three parallel speciation events in three different lakes of the area (Rundle 2000). ${ }^{12}$

In addition to these biological reasons, there is another flaw in the proposed criteria. If species membership can be defined exclusively by the relations it bears with other individuals, it is conceptually impossible to determine the species of a hypothetical organism that is the only one of its species. Let us imagine that Sgrunt is the unique specimen of a kind of living being, a sort of monstrum. Now, Sgrunt does not exist, and very probably, mono-species organisms have never lived. Moreover, according to working biologists, it is simply meaningless to affirm that there exists a species with just one example. However, from the metaphysical and conceptual point of view, it would seem possible to ascribe a species to Sgrunt. In the end, it has a series of features that characterize it. But the definitional criteria of the species-as-individuals approach block a priori the possibility to collocate Sgrunt into a species, and this is suspicious.

If an organism cannot belong to a species solely by virtue of its relations with other individuals, it does belong to a species because of certain intrinsic features. But this is precisely what the species-as-kinds advocates state - it is because an organism has certain properties (i.e. it is an instance of a certain kind) that it is a member of a species. The insufficiency of relational criteria undermines the basis of the conception of species as complex individuals, since it pushes towards the view that living beings are collected in species not because of their relationships but by virtue of having certain features. 


\section{A conceptualist model for the metaphysics of species}

In a series of articles (2000, 2001), Barry Smith introduced an important distinction between, roughly, fiat and bona fide entities. In particular, he distinguishes between fiat boundaries, which are the outcome of an individual or collective intentional act, and bona fide boundaries, which are (in a sense) grounded in the reality of things. There is no doubt that political boundaries (for instance, those of some states of the United States) are fiat, while the boundaries of an object like a table seem to be bona fide. Behind this distinction, there is the old-as-philosophy topic of carving the nature at joints, that is, the question of realism. Achille Varzi (2001), for instance, maintained a strong conventionalist position: there are no bona fide boundaries, and thus, there are no bona fide objects. This debate is also relevant for our analysis.

Barry Smith (2001, p. 34) states that biological species are a typical example of fiat objects. We believe that two different philosophical theses are condensed in this view: the first has a metaphysical character and concerns the nature of the entities under scrutiny; the second has an ontological character and concerns the existence and modality of existence of the entities in question. Therefore, according to the first subclaim, biological species are individuals, that is, spatiotemporally collocated items. Moreover, the species are scattered, not connected, individuals. The second subclaim - the proper 'fiat' part - has to do with the ontological dependence of species. If they are fiat entities, they strongly depend on cognitive human acts. In a nutshell, a world with no intentionality is a world with no biological species.

Now, if the arguments we advanced above are correct, Smith's thesis in which biological species are fiat objects cannot be entirely endorsed. We argued that there are good reasons to believe that species are not individual entities. ${ }^{13}$ However, the problems that stem from evolutionary theory and cast doubt on the alleged identification of biological species with natural kinds still persist. We arrive at a sort of stalemate. On one hand, there are good reasons not to consider species as individuals. On the other, even if universals, species seem to show no necessary and fixed features as the natural kinds. In the following, we will sketch a solution that tries to unify both a realist intuition (departing from Varzi-Smith's conventionalism) and the data from biology.

We will assume that in the world there exist at least some biological properties that are bona fide entities; a nominalist would disagree, of course. But it is meaningful, from a philosophical point of view, that the very biologists advancing the species-as-individuals view admit the existence of biological 
properties and relations. ${ }^{14}$ Any living organism exemplifies certain clusters of properties, intended as bona fide entities. Now, an advocate of the speciesas-kind view could identify some privileged clusters with natural kinds. This manoeuver bumps into the well-known problems we previously discussed. Our idea is to exploit the distinction between fiat and bona fide by applying it not to individuals or properties but to concepts. Smith (2001, pp. 141-2) discusses fiat concepts. We wish to extend his considerations to the metaphysics of species. We assume that concepts are epistemic entities (they are what we understand), and in this sense, they are dependent on the human mind. Classically, concepts can be individual (for instance, the concept associated with the proper name Kurt), universal (for instance, concepts expressed by predicates such as 'is white' or 'is a cat') and, finally, logical (as those expressed by particles such as 'or', 'and' or 'not'). The ontological tie between universal concepts and individuals is not instantiation but falling under. One could state that the reason Socrates falls under the concept of being wise is that Socrates instantiates the property (if any) of wisdom. Universal concepts are the clippings by which the world can be categorized. In Smith's words,

Suppose that each concept is associated with some extended region in which its actual and possible instances are contained, and suppose further that this is done in such a fashion that the prototypes, the most typical instances, are located at distances in the center in proportion to their degree of non-typicality. (Smith 2001, p. 141)

Now, Smith's view is that concepts are typically fiat entities, since they depend on the intentional acts of the subjects. This is true, but it grasps just one side of our proposal. What we want to maintain is that biological species are universal concepts and that they are grounded in the biological nature of the organisms. A very simplified example follows: let us imagine that there are just three biological properties $(P, Q, R)$ that come up as graduated (they are determinable properties). For convenience's sake, we will indicate the measure of the property under consideration with a number in the interval $1-1,000$. Moreover, let us imagine that in our domain, we have just three individuals $(a, b, c)$ that exemplify these properties and we have to classify them in species. The first scenario we consider is the following:

$\begin{array}{lllll}a & : & P_{235} & Q_{28} & R_{177} \\ b & : & P_{233} & Q_{28} & R_{176} \\ c & : & P_{236} & Q_{27} & R_{177}\end{array}$


Given this scenario, it is wholly plausible to consider the three individuals as belonging to the same species. ${ }^{15}$ Thus, we will say that $a, b$ and $c$ fall under species $S$ because it is a fact of the matter that they instantiate a significant cluster of biological properties.

Unfortunately for working biologists, things are very rarely so easy. Let us consider another scenario:

$\begin{array}{lllll}a & : & P_{670} & Q_{750} & R_{800} \\ b & : & P_{668} & Q_{751} & R_{20} \\ c & : & P_{31} & Q_{750} & R_{802}\end{array}$

What happens in this case? Clearly, based on these data, $a, b$ and $c$ cannot be subsumed under the same concept of species. Indeed, we have that

$a$ and $c$ are different concerning property $P$.

$a$ and $b$ are different concerning property $R$.

$b$ and $c$ are different concerning properties $P$ and $R$.

Now, we can notice the typical fiat feature of the concept of species - it is a biologist's choice to decide what the most relevant properties are. They could, for instance, maintain that property $P$ is, above all, less important and then consider $a$ and $c$ as belonging to the same species. Alternatively, they can reason the same way about $R$. Another possibility is to focus on property $Q$ and then declare that $a$ and $b$ and $c$ belong to the same species, their differences notwithstanding. There are so many available alternatives open to biologists to categorize this scenario. However - and this is the trait that differentiates the world of living beings from, say, the world of geography - not all the divisions are possible. In this case, it is quite unreasonable to consider $b$ and $c$ to belong to a species (say, $S$ ) and $a$ not to belong to $S$. And this is because it is a fact of the matter that some clusters of properties are instantiated and others are not. It is plausible to think that in cases such as the political boundaries between Wyoming and Colorado, there are no choices that must be disregarded in principle.

The toy model we have provided can easily be extended to a much higher number of properties. In particular, we can represent any determinable property as a dimension. So we get an $n$-dimensional ${ }^{16}$ space, where $n$ indicates the number of properties taken into account.

In our framework, if we model $P, Q$ and $R$ as dimensions, we obtain a tridimensional space in which we can place the individuals. Each living being instantiates the properties $P, Q$ and $R$ to a certain degree; this means that in 
the model, any organism occupies a certain position in the space. Very close organisms constitute clouds in the space. It is now clear why the concepts are seen as clippings - it is natural to consider close individuals as belonging to the same species, while it is odd to make partitions of the space that includes nonhomogeneous regions, empty spaces or even disconnected areas.

Nevertheless, there are doubtful cases. For example, some individuals can be far from the centre of a cloud; they are borderline cases. In this scenario, biologists have to decide whether to cut the space with a concept of species that includes the case under scrutiny or consider it as belonging to another species. Then, although they are grounded in re, the concepts of species are vague.

Let us see how our framework accounts for the evolutionary phenomena. From a merely ontological point of view, what mutates from generation to generation is the exemplification of some biological properties (think about determinable properties as, for example, 'having an olfactory system that is sensitive to a certain kind of pollen'). With the passage of time, the conceptual clipboard, that is, the species Felis Catus, no longer encompasses the majority of the descendants that now instantiate different properties. Therefore, it is natural to select the cluster of individuals in a different way. The origin of a new species is precisely the acknowledgement, on a conceptual level, of a new clipping in the space of biological properties and individuals. Analogously, extinct species, such as Tyrannosaurus Rex, are concepts under which no living organisms now fall. They are classifications of properties with no instances. But, if in a Jurassic Park laboratory, a scientist created animals 'in vitro' that exemplify the cluster of properties subsumed under the species Tyrannosaurus Rex, these animals are really members of the species in question. A similar consideration could be made for the hypothetical case of alien species.

\section{Conclusion}

In this chapter, we discussed the metaphysical interpretation of the concept of biological species. One of the reasons to adopt the species-as-individuals view is the preference for relational species criteria over structural ones. However, we have shown that relational criteria provide neither necessary nor sufficient conditions for species membership. In order to group biological individuals in taxa, we need to use structural properties and the similarity among individuals. 
Thus, one of the main arguments in favour of the species-as-individuals view fails. Then, we provided a framework to consider biological species as concepts instead of individuals or natural kinds. These concepts show both fiat and bona fide features. On one hand, they depend on the real instantiation of biological properties by living beings. However, on the other hand, they reflect the decisions made by biologists when evaluating the importance of properties or considering borderline cases. We argued that this model can overcome some difficulties that affect the previous views.

\section{Notes}

1 In Chapter 6 of this volume, Vogt considers biological entities as 'causal agents in various biological processes'. Among them, reproduction and evolution are important in defining their identity.

2 For the modern biological concept of species, see Dobzhansky (1935), Dobzhansky (1937), Mayr (1942), Mayr (1949) and Mayr (1970).

3 Therefore, this view mixes elements of the phylogenetic concept of species with elements of the biological concept. Compare Henning (1966).

4 These phylogenetic concepts, which are grounded on traits that are not present in the ancestors of a population, mix elements of the phylogenetic concept of species with elements of the classical morphological concept as a set of individuals that instantiate some properties. Compare Rosen (1978), Eldredge and Cracraft (1980) and Nelson and Plamick (1981).

5 It is important to note that the biological and phylogenetic concepts of species do not have the species-as-individuals view as a necessary consequence. In fact, the species-as-kinds views that hold relations among members as a criterion of membership are also possible (cf. see Okasha 2002 and LaPorte 2004). However, it should be noted that, if the membership criterion is based on relations among members, it is much more natural to consider species as complex individuals whose cohesion is a result of the relations among the organisms that are parts of the species (for this thesis, cf. Crane 2004).

6 Mayr (1991) defines a species as 'a reproductively isolated aggregate of populations which can interbreed with one another because they share the same isolating mechanisms' (p. 186).

7 Templeton (1989) affirms that hybridization is not rare even among animals, as his study on mammals would show.

8 Some proposals aim even at questioning Linneaus's hierarchy and to substitute it with a classification in which every taxon is monophyletic (cf. de Queiroz and Gauthier 1992, Ereshefsky 2001). 
9 As it often happens with biological species, one can draw an analogy between this example and an example concerning languages. Suppose that on a very faraway planet a population speaks a language indistinguishable from English (same phonology, morphology, lexicon, grammar, etc.). Suppose that terrestrial English and the English of this population are not genetically linked, but evolved independently. Are terrestrial English and the twin English the same language or two different languages? Suppose, for example, that this population moves on Earth and mixes with English speakers. Is it possible to hold that the two populations speak two different languages similar in every aspect?

10 For a criticism of the notion of thought in biology and philosophy of biology, compare Hull (1989).

11 For an argument along these lines, which uses allopolyploid species, compare Stamos (2003, pp. 237-9).

12 Vogt (see Chapter 6 of this volume) maintains that common historical origin can serve as a principle of connectedness that allows the delineation of bona fide objects. However, if the criticisms advanced here are corrected, species cannot be considered bona fide individuals on the basis of a common historical origin. Another reason why species might be seen as bona fide individuals according to Vogt's criteria is that they are often considered as the units of evolution, what might be described as 'the engines of evolution' (Eldredge and Cracraft 1980; Wiley 1981; Mayr 1982; Ghiselin 1987). Thus, their unity might be causal or functional. However, that species are evolutionary units is a controversial claim. Molecules, genes, cells, organisms, populations have been proposed as possible units of evolutions (cf. Brandon and Burian 1986 for discussions on this topic) and it is not clear which are the levels at which selection acts. For a criticism of the idea that species are evolutionary units, compare Ereshefsky (1991).

13 Obviously, there is a sense in which we can non-ambiguously refer to a biological species by meaning the individuals of that species collectively considered. But the problem is metaphysical - does that species coincide with the conglomeration of individuals?

14 Tuomas Tahko (2012) argues contra Varzi and Smith that fundamental natural laws suggest the existence of bona fide entities. Tahko's examples have to do with quantum physics, and in particular, with the properties of fundamental particles. It is an open question if the same considerations can also hold for levels of reality as complex as those of living beings.

15 In our example, the differences among individuals are graduated using an interval $1-1,000$. There is no reason to prefer this level of distinction to one higher (or lower). In practice, this choice also crucially depends on the fine-tuning of the instruments.

16 Compare Gardenfors (2000). 


\section{References}

Brandon, R. N. and Burian, R. (eds) (1986), Genes, Organisms, Populations.

Controversies over the Units of Selection, MIT Press, Cambridge, MA.

Burger, W. (1975). 'The Species Concept in Quercus', Taxon, 24: 45-50.

Crane, J. (2004), 'On the Metaphysics of Species', Philosophy of Science, 71: 156-73.

Dobzhansky, T. (1935), 'A Critique of the Species Concept in Biology', Philosophy of Science, 2 (3): 344-55.

Dobzhansky, T. (1937), Genetics and the Origin of Species, Columbia University Press, New York.

Ehrlich, P. and Raven, P. (1969), 'Differentiation of Populations', Science, 165: 1228-32.

Eldredge, N. and Cracraft, J. (1980), Phylogenetic Patterns and the Evolutionary Process, Columbia University Press, New York.

Ereshefsky, M. (1991), 'Species, Higher Taxa, and the Units of Evolution', Philosophy of Science, 58 (1): 84-101.

Ereshefsky, M. (2001), The Poverty of the Linnaean Hierarchy: A Philosophical Study of Biological Taxonomy, Cambridge University Press, Cambridge.

De Queiroz, K. and Gauthier, J. (1992), 'Phylogenetic Taxonomy', Annual Review of Ecology and Systematics (23): 449-80.

Gärdenfors, P. (2000), Conceptual Spaces: The Geometry of Thought, MIT Press, Cambridge, MA.

Ghiselin, M. (1966), 'On Psychologism in the Logic of Taxonomic Controversies', Systematic Zoology, 15: 207-15.

Ghiselin, M. (1974), 'A Radical Solution to the Species Problem', Systematic Zoology, 23 (4): 536-44.

Ghiselin, M. (1987), 'Species Concepts, Individuality, and Objectivity', Biology \& Philosophy, 2: 127-43.

Henning, W. (1966), Phylogenetic Systematics, University of Chicago Press, Chicago.

Hull, D. (1965a), 'The Effect of Essentialism on Taxonomy-Two Thousand Years of

Stasis (I)', The British Journal for the Philosophy of Science, 15 (60): 314-26.

Hull, D. (1965b), 'The Effect of Essentialism on Taxonomy-Two Thousand Years of

Stasis (II)', The British Journal for the Philosophy of Science, 16 (61): 1-18.

Hull, D. (1976), 'Are Species Really Individuals?', Systematic Zoology, 25 (2): 174-91.

Hull, D. (1978), 'A Matter of Individuality', Philosophy of Science, 45 (3): 335-60.

Hull, D. (1989), 'A Function for Actual Examples in Philosophy of Science', in M. Ruse (ed.) What the Philosophy of Biology Is, Kluwer, Dordrecht, 309-21.

Irwin, D., Bensch, S. and Price, T. (2001), 'Speciation in a Ring', Nature, 409: 333-7.

LaPorte, J. (2004), Natural Kinds and Conceptual Change, Cambridge University Press, Cambridge.

Lowe, E. J. (1998), The Possibility of Metaphysics: Substance, Identity, and Time, Oxford University Press, Oxford. 
Mayr, E. (1942), Systematics and the Origin of Species, Columbia University Press, New York.

Mayr, E. (1949), 'The Species Concept: Semantics Versus Semantics', Evolution, 3: 371-2. Mayr, E. (1970), Populations, Species, and Evolution, Harvard University Press, Cambridge. Mayr, E. (1982), The Growth of Biological Thought, Harvard University Press, Cambridge. Mayr, E. (1987), 'On the Ontological Status of Species: Scientific Progress and Philosophical Terminology', Biology and Philosophy, 2: 145-66.

Mayr, E. (1991), One Long Argument, Harvard University Press, Cambridge. Nelson, G. and Plamick, N. (1981), Systematics and Biogeography: Cladistics and Vicariance, Columbia University Press, New York.

Okasha, S. (2002), 'Darwinian Metaphysics: Species and the Question of Essentialism', Synthese, 131: 191-213.

Ridley, M. (1993), Evolution, Blackwell Scientific Publications, Boston.

Rosen, D. (1978), 'Vicariant Patterns and Historical Explanation in Biogeography', Systematic Zoology, 27: 159-88.

Rundle, H. et al. (2000), 'Natural Selection and Parallel Speciation in Sympatric Sticklebacks', Science, 287: 306-8.

Simons, P. (2013), 'Vague Kinds and Biological Nominalism', Metaphysica, 14: 275-82.

Smith B. (1999), 'Agglomerations', in C. Freksa and David M. Mark (eds), Spatial Information Theory. Cognitive and Computational Foundations of Geographic Information Science (Springer Lecture Notes in Computer Science 1661), 1999, 267-82.

Smith, B. (2001), 'Fiat Objects', Topoi, 20 (2): 131-48.

Smith, B. and Varzi, A. C. (2000), 'Fiat and Bona Fide Boundaries', Philosophy and Phenomenological Research, 60: 401-20.

Sober, E. (1980), 'Evolution, Population Thinking, and Essentialism', Philosophy of Science, 47 (3): 350-83.

Stamos, D. (2003), The Species Problem, Lexington Books, Oxford.

Tahko, T. E. (2012), 'Boundaries in Reality', Ratio, 25 (4): 405-24.

Templeton, A. (1989), 'The Meaning of Species and Speciation. A Genetic Perspective', in D. Otte and J. Endler (eds), Speciation and its Consequences, Sinauer associates, Sunderland.

Vrba, E. (1985), 'Introductory Comments on Species and Speciation', in E. Vrba, (ed.),

Species and Speciation, Transvaal Museum, Pretoria, ix-xviii

Van Valen, L. (1976), 'Ecological Species, Multispecies, and Oaks', Taxon, 25: 233-9.

Varzi, A. (2001), 'Vagueness in Geography', Philosophy and Geography, 4: 49-65.

Wiley, E. (1981), Phylogenetics: The Theory and Practice of Phylogenetic Systematics,

Wiley and Sons, New York. 